\title{
Evaluación de la musculatura inspiratoria en niños con antecedentes de Mielomeningocele
}

\author{
DANIEL ZENTENO A. ${ }^{1}$, HOMERO PUPPO G. ${ }^{2}$, RAMIRO GONZÁLEZ V. ${ }^{3}$, \\ ROBERTO VERA U. ${ }^{4}$, RODRIGO TORRES C. ${ }^{4}$, KUO HO CHUNG-YANG ${ }^{4}$, \\ DOLORES PAVÓN T. ${ }^{3}$, M. ANGÉLICA PÉREZ H. ${ }^{3}$, GUIDO GIRARDI B. ${ }^{3}$
}

1. Pediatra. Becado de Neumología, Departamento de Pediatría Sur, Facultad de Medicina, Universidad de Chile. Unidad de Broncopulmonar, Hospital Exequiel González Cortés, Santiago, Chile.

2. Kinesiólogo, Departamento de Pediatría Sur, Facultad de Medicina, Universidad de Chile. Unidad de Broncopulmonar, Hospital Exequiel González Cortés, Santiago, Chile.

3. Pediatra Broncopulmonar, Departamento de Pediatría Sur, Facultad de Medicina, Universidad de Chile. Unidad de Broncopulmonar, Hospital Exequiel González Cortés, Santiago, Chile.

4. Kinesiólogo, Programa Nacional de Ventilación Mecánica No-Invasiva, Santiago, Chile.

\begin{abstract}
Evaluation of inspiratory musculature in children with myelomeningocele

Background: Respiratory musculature function in patients with myelomeningocele (MMC) has not been evaluated sufficiently. Impairment in inspiratory muscles capacity could make patients prone to complications in clinical situations in which the respiratory work is increased. Objective: Evaluate inspiratory muscle function, measured with Maximal Inspiratory Pressure (MIP) in children with MMC. Method: In 13 children with MMC, MIP was obtained according to Black and Hyatt technique, using an aneroid gauge pressure. The results were compared to Szeinberg reference values by $t$ Student, where $p<0.05$ was considered significant. Results: The average age was $11.9 \pm 2.8$ years and 10 female. Expected MIP average was $112 \pm$ $20 \mathrm{cmH}_{2} \mathrm{O}$, with lower limit of $92 \mathrm{cmH}_{2} \mathrm{O}$. The observed Pimax was $62 \pm 23 \mathrm{cmH}_{2} \mathrm{O}, 33 \%$ less than the inferior limit expected $(\mathrm{p}<0.05)$. Conclusions: These children present a decrease of inspiratory musculature force, making them suceptible to muscle fatigue in pathological conditions, requiring a greater respiratory effort and difficulties in mechanical ventilation weaning. We suggest that a regular study of inspiratory muscles is neccessary in MMC patients, in order to define a respiratory musculature training necessity.

(Key words: Maximal Inspiratory Pressure, pulmonary function, myelomeningocele).

Rev Chil Pediatr 2008; 79 (1): 21-25
\end{abstract}

\section{RESUMEN}

Introducción: Existe escasa evidencia que evalúe la función muscular respiratoria de niños con antecedente de mielomeningocele (MMC). La disfunción de la musculatura inspiratoria puede facilitar complicacio-

Trabajo recibido el 10 de mayo de 2007, devuelto para corregir el 26 de julio de 2007, segunda versión el 20 de septiembre de 2007, aceptado para publicación el 14 de noviembre de 2007.

Correspondencia a:

Daniel Zenteno A.

E-mail: danielzenteno@gmail.com 
nes respiratorias. Objetivo: Evaluar la función de la musculatura inspiratoria, medida a través de la Presión inspiratoria máxima (Pimax), en niños con antecedente de MMC. Pacientes y Métodos: En 13 niños se obtuvo Pimax mediante técnica de Black and Hyatt, utilizando un manómetro aneroide. Los valores obtenidos fueron comparados con los valores de referencia de Szeinberg utilizando t Student, considerando significativos p < 0,05. Resultados: La mediana de edad fue 11 años (rango: 8-18; 10 mujeres). La Pimax promedio esperada fue $112 \pm 20 \mathrm{cmH}_{2} \mathrm{O}$ (límite inferior $92 \mathrm{cmH}_{2} \mathrm{O}$ ) mientras la Pimax observada fue $62 \pm 23$ $\mathrm{cmH}_{2} \mathrm{O}$, siendo $33 \%$ menor que el límite inferior del valor esperado ( $\left.<<0,05\right)$. Discusión: Los niños con antecedente de MMC presentaron disminución de la fuerza de los músculos inspiratorios que los podría hacer más susceptibles a la fatiga muscular en situaciones que impliquen mayor trabajo respiratorio así como dificultades frente a la desconexión al ser sometidos a ventilación mecánica.

(Palabras clave: Presión inspiratoria máxima, función pulmonar, mielomeningocele).

Rev Chil Pediatr 2008; 79 (1): 21-25

\section{Introducción}

El Mielomeningocele (MMC) es un defecto congénito de los arcos vertebrales con dilatación quística de las meninges, anormalidades estructurales y funcionales de la médula espinal o la cauda equina ${ }^{1}$. En países desarrollados las malformaciones del tubo neural son la segunda causa de malformaciones congénitas (MC) luego de las cardiopatías congénitas, siendo el MMC la principal causa de MC del tubo neural ${ }^{2}$. Su incidencia es variable en distintos países y regiones; países desarrollados poseen una incidencia de 0,7-2 casos por 1000 recién nacidos vivos (RNV) al año donde se incluye Chile. En algunos países latinoamericanos su incidencia es cercana a 12 por $1000 \mathrm{RNV}^{1,2}$.

En estas 3-4 últimas décadas, su incidencia ha disminuido significativamente, tanto a nivel nacional e internacional, gracias al éxito del aporte preconcepcional de ácido fólico ${ }^{3-5}$. Pese a que su corrección quirúrgica tradicionalmente se realiza en los primeros días de vida ${ }^{6}$, los pacientes con MMC desarrollan con frecuencia numerosas secuelas, en especial neurológicas, ortopédicas, urológicas y gastrointestinales. A su vez, las complicaciones neurológicas y ortopédicas como invalidez parcial o total, escoliosis y obesidad ${ }^{7-9}$ pueden influir en su función pulmonar.

Los estudios sobre función pulmonar en estos pacientes son limitados y controversiales ${ }^{10,11}$. Según Sherman y colaboradores, encontraron disminución de la capacidad inspiratoria ${ }^{10}$; sin embargo, Swaminthan y colaboradores no en- contraron esta relación ${ }^{11}$. La capacidad inspiratoria ha demostrado poder ser entrenada y mejorada en algunas patologías, tanto en niños como en adultos ${ }^{12-14}$; generando un efecto positivo en la calidad de vida y disminución de complicaciones especialmente postoperatorias ${ }^{15,16}$. El objetivo del presente estudio fue evaluar la fuerza de la musculatura inspiratoria, medida a través de presión inspiratoria máxima (Pimax), en niños con antecedentes de MMC, comparado con los valores de referencia según edad.

\section{Pacientes y Métodos}

Se reclutaron 13 niños escolares y adolescentes, con antecedentes de MMC operados durante sus primeros días de vida, que constituían la totalidad de niños colaboradores y controlados por esta patología, durante el periodo Enero 2004 a Mayo 2006, en las Unidades de Neurología y Broncopulmonar del Hospital de niños Exequiel González Cortés. Se excluyeron pacientes con antecedentes de patología respiratoria crónica o recurrente y patología respiratoria aguda significativa que hubiesen requerido de manejo intrahospitalario.

En condiciones basales, luego de su adiestramiento (educación de la técnica), se realizó medición de Pimax a partir de volumen residual, mediante la técnica de Black and Hyatt ${ }^{17}$ utilizando un manómetro aneroide DHD 550120 (New York, USA) calibrado en centímetros de agua ( 0 a $\left.-120 \mathrm{cmH}_{2} \mathrm{O}\right)$. Los valores obtenidos fueron comparados con los límites infe- 
Tabla 1. Valores de referencia esperados según edad y género. Szeinberg y cols ${ }^{18}$

\begin{tabular}{|c|c|c|c|}
\hline Edad & $\begin{array}{l}\text { Talla } \\
\text { (cms) }\end{array}$ & $\underset{\left(\mathrm{cm} \mathrm{H} \mathrm{H}_{2} \mathrm{O}\right)}{\operatorname{Pimax}}$ & $\begin{array}{c}\text { Pimax lim } \\
\text { inferior } \\
\left(\mathrm{cm} \mathrm{H}_{2} \mathrm{O}\right)\end{array}$ \\
\hline \multicolumn{4}{|l|}{ Varones } \\
\hline $8-10,9$ & $136 \pm 7$ & $116 \pm 26$ & 90 \\
\hline $11-13,9$ & $151 \pm 10$ & $130 \pm 16$ & 114 \\
\hline $14-16,9$ & $172 \pm-8$ & $126 \pm 22$ & 104 \\
\hline $17-20,9$ & $179 \pm-6$ & $143 \pm 12$ & 131 \\
\hline $21-40$ & $180 \pm-7$ & $123 \pm 12$ & 111 \\
\hline \multicolumn{4}{|l|}{ Mujeres } \\
\hline $8-10,9$ & $139 \pm 7$ & $104 \pm 20$ & 84 \\
\hline $11-13,9$ & $154 \pm 7$ & $112 \pm 20$ & 92 \\
\hline $14-16,9$ & $162 \pm 6$ & $109 \pm 21$ & 88 \\
\hline $17-20,9$ & $164 \pm 7$ & $107 \pm 25$ & 82 \\
\hline $21-40$ & $163 \pm 8$ & $91 \pm 20$ & 71 \\
\hline
\end{tabular}

riores de los valores de referencia de Szeinberg y colaboradores ${ }^{18}$ (tabla 1) según edad y género, utilizando t Student para el análisis estadístico y considerando significativos $\mathrm{p}<0,05$.

\section{Resultados}

La mediana de edad de los niños fue 11 años (rango: 8-18); 10 mujeres. Todos los sujetos presentaban distintos grados de escoliosis, siendo el Angulo de Cobb promedio de 76 grados (rango: 42-142); 12/13 eran portadores de vejiga neurogénica, 4/13 de intestino neurogénico, 3/13 infecciones urinarias recurrentes, 5/13 alérgicos al látex y 3/13 obesos. Presentaban algún grado de invalidez 11/13 menores y

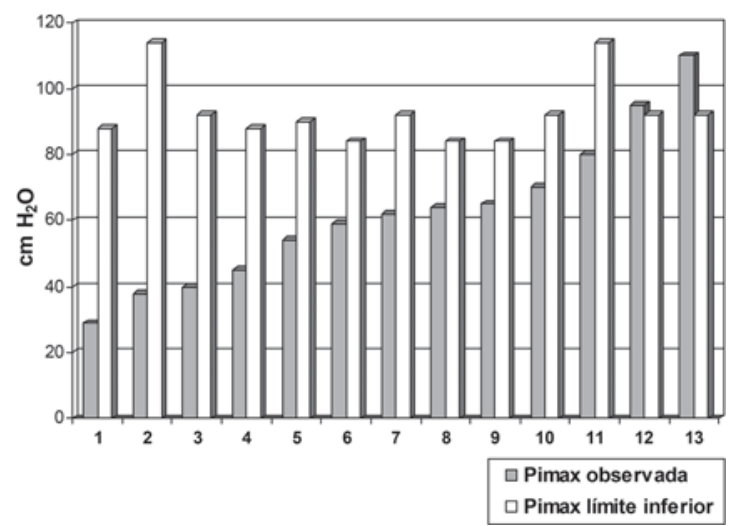

Figura 1. Valores de Pimax observados vs Pimax límite inferior en los 13 pacientes. sólo 2/13 eran autovalentes.

Según valores de referencia de Gutiérrez y colaboradores $^{20}$ las espirometrías de los pacientes mostraron: 5/13 alteración ventilatoria restrictiva (AVR) avanzada, 3/13 moderada y 3/13 leve; sólo 2/13 presentaban espirometrías normales. Los valores espirométricos promedios y DE, considerando la envergadura ${ }^{19}$, eran: CVF (capacidad vital forzada) $58 \pm 18 \%, \mathrm{VEF}_{1}$ (volumen espiratorio forzado al primer segundo) $58 \pm 17 \%, \mathrm{VEF}_{1} / \mathrm{CVF}$ (relación entre $\mathrm{VEF}_{1}$

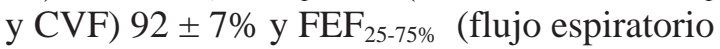
forzado al 25-75\% de la CVF) de $64 \pm 22 \%$. Ocho niños tenían datos de Ventilación Máxima Voluntaria (VMV), 3 de ellos presentaron valores disminuidos, los que a su vez tenían espirometrías con alteraciones ventilatorias restrictivas de grado avanzado.

La figura 1 muestra los valores de Pimax observados vs Pimax esperada límite inferior en los 13 pacientes, en orden creciente según Pimax observada. Se aprecia que sólo 2/13 niños presentaron Pimax dentro del rango normal o mayor al límite inferior esperado. En el resto de los niños 11/13, la Pimax fue menor a este límite. Se destaca que 6/13 tenían Pimax bajo $60 \mathrm{~cm} \mathrm{H}_{2} \mathrm{O}$. La Pimax promedio esperada fue $112 \pm 20 \mathrm{cmH}_{2} \mathrm{O}$, siendo el límite inferior promedio esperado de $92+10 \mathrm{cmH}_{2} \mathrm{O}$; sin embargo, la Pimax obtenida promedio fue sólo de $62 \pm 23 \mathrm{cmH}_{2} \mathrm{O}$ (figura 2). Esta diferencia entre los valores observados y los límites inferiores de los valores de referencia fue 33\% menor $(\mathrm{p}<0,05)$.

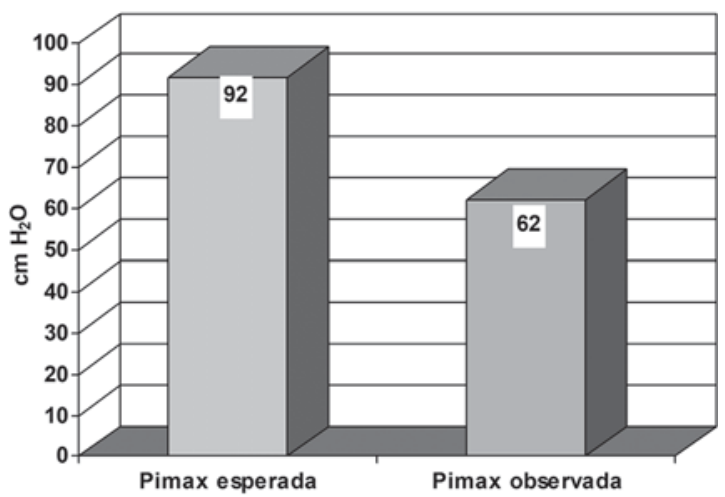

Figura 2. Límite inferior de Pimax esperada vs Pimax observada en los 13 pacientes. 


\section{Discusión}

Nuestro estudio demuestra, que en los niños con antecedentes de MMC evaluados, existió una disminución de la capacidad inspiratoria evaluada mediante Pimax. Según los valores de referencia de Szeinberg y colaboradores ${ }^{18}, 11 / 13$ demostraron una Pimax bajo el límite inferior esperado para su edad y sexo; 6/13 presentaban Pimax bajo $60 \mathrm{cmH}_{2} \mathrm{O}$ considerada por Lotters y colaboradores, como límite para hablar con propiedad de debilidad de la musculatura inspiratoria ${ }^{12,21}$. Esto apoya los resultados de Sherman y colaboradores ${ }^{10}$ que encontró una reducción de Pimax bajo $60 \mathrm{~cm}$ de $\mathrm{H}_{2} \mathrm{O}$ en el 33\% de los niños estudiados con MMC.

En los 8 niños que se logró evaluar VMV, menos de la mitad (3/8) presentaron valores disminuidos con respecto a los valores esperados; estos a su vez poseían CVF disminuida en grado avanzado y Pimax disminuida.

Los niños presentaban similares antecedentes mórbidos y/o secuelas que en otras series $^{2,7,8,9,22,23}$, sin embargo, existía un mayor porcentaje de escoliosis (100\%) y de invalidez $(85 \%)^{2,7,8,9,22,23}$, lo que podría explicar en cierta forma el porcentaje marginalmente mayor de Pimax demostrada en el estudio de Sherman y colaboradores ${ }^{10}$. Los estudios sobre función pulmonar en pacientes con MMC son muy limitados, en especial cuando se hace mención a la capacidad inspiratoria ${ }^{10,11}$. Los casos analizados son de 12 a 14, nosotros enrolamos un número similar de sujetos. Este reducido número de casos no nos permite realizar otros análisis estadísticos confiables; por otro lado, la colaboración no siempre es posible por sus alteraciones neuromusculares y mentales ${ }^{22}$. Debemos considerar que no es una patología que con frecuencia se le de importancia a las consecuencias sobre el sistema respiratorio ${ }^{2}$ hasta el momento en que se toman decisiones como corrección quirúrgica de escoliosis, lo que explica que todos nuestros niños presentaran algún grado de escoliosis cuando al momento de evaluar la Pimax.

Una de las complicaciones más frecuentes de las correcciones quirúrgicas de escoliosis es la ventilación mecánica prolongada. Yuan y colaboradores demostraron diversos factores relacionados con requerimientos de ventilación mecánica mayor o igual de 3 días donde se encontraban la enfermedad neuromuscular y la Pimax inferior a la esperada ${ }^{16}$. Dentro de las enfermedades neuromusculares se incluyen niños con antecedentes de MMC. La Pimax es un factor potencialmente entrenable, lo cual está ampliamente demostrado en adultos con patología pulmonar obstructiva crónica ${ }^{12}$ e incluso con patologías pulmonares restrictivas ${ }^{13}$. En niños el entrenamiento de la musculatura inspiratoria ha sido beneficioso en patologías en enfermedades neuromusculares como Enfermedad de Duchenne y atrofia espinal tipo III ${ }^{24,25}$, así como también en fibrosis quística ${ }^{14}$.

De los 11 niños con Pimax disminuida, en 7 casos se realizó entrenamiento de la musculatura inspiratoria durante un periodo de 6 a 8 semanas. La mayoría de ellos (6/7) logró Pimax sobre $60 \mathrm{~cm}$ de $\mathrm{H}_{2} \mathrm{O}$ y 4 normalizaron valores, es decir, alcanzaron Pimax sobre el límite interior del valor esperado para su edad y sexo. Actualmente, nuestro equipo está trabajando en el entrenamiento de la musculatura respiratoria de niños con antecedentes de MMC, como también en menores portadores de otras enfermedades neuromusculares y respiratorias crónicas, con el objetivo de evaluar la repercusión sobre su calidad de vida, en su vida cotidiana y el efecto sobre complicaciones postoperatorias.

En conclusión, los niños con MMC estudiados presentaban una menor fuerza de los músculos inspiratorios; esto, los puede hacer más proclives a la fatiga muscular respiratoria y a la posibilidad de destete dificultoso cuando sean sometidos a ventilación mecánica. Frente a la evidencia presentada, sugerimos el estudio sistemático de los músculos inspiratorios en estos pacientes para determinar un eventual entrenamiento muscular respiratorio, especialmente si el paciente va ser sometido a una cirugía programada.

\section{Agradecimientos}

A los Médicos, Kinesiólogos y Personal de nuestra Unidad de Broncopulmonar y Laboratorio de Función Pulmonar que día a día atienden a estos niños. Finalmente, a nuestros pa- 
cientes, que gracias a su colaboración permiten concretar este trabajo.

\section{Referencias}

1.- Detrait ER, George TM, Etchevers HC, Gilbert JR, Vekemans $M$, Speer MC: Human neural tube defects: developmental biology, epidemiology, and genetics. Neurotoxicol Teratol 2005; 27: 515-24.

2.- Kolaski K: Myelomeningocele. Emedicine Journal [revista electrónica]. April 2006 [consultado 06-04-2007]: Disponible en http://www.emedicine.com/pmr/topic83. html

3.- Pitkin RM: Folate and neural tube defects. Am J Clin 2007; 85 (Supl): 285-88.

4.- Eichholzer $M$, Tonz O, Zimmermann R: Folic acid: a public-health challenge. Lancet 2006; 22 (367): 135261.

5.- Hertrampf E, Cortés F: Folic acid fortification of wheat flour: Chile. Nutr Rev 2004; 62: 44-8.

6.- Matuszczak E, Lenkiewicz T: Results of surgical treatment of myelomeningocele. Eur J Pediatr Surg 2006; 16: 337-42.

7.- Asher $M$, Olson J: Factors affecting the ambulatory status of patients with spina bifida cystica. J Bone Joint Surg 1983; 65: 350-6.

8.- Locke MD, Sarwark JF: Orthopedic aspects of myelodysplasia in children. Curr Opin Pediatr 1996; 8: 657.

9.- Mita K, Akataki K, Itoh K, et al: Assessment of obesity of children with spina bifida. Dev Med Child Neurol 1993; 35: 305-11.

10.- Sherman MS, Kaplan JM, Effgen S, Campbell D, Dold F: Pulmonary dysfunction and reduced exercise capacity in patients with myelomeningocele. J Pediatr 1997; 131: 413-8.

11.- Swaminathan S, Paton JY, Ward SL, Jacobs RA, Sargent CW, Keens TG: Abnormal control of ventilation in adolescents with myelodysplasia. J Pediatr 1989; 115: 898-903.

12.- Lotters F, Van Tol B, Kwakkel G, Gosselink R: Effects of controlled inspiratory muscle training in patients with COPD: a meta-analysis. Eur Respir J 2002; 20: 570-6.

13.- Budweiser S, Moertl M, Jorres RA, Windisch $W$, Heinemann F, Pfeifer M: Respiratory muscle training in restrictive thoracic disease: a randomized controlled trial. Phys Arch Med Rehabil 2006; 87: 1559-65.

14.- De Jong W, Van Aalderen WM, Kraan J, Koeter GH, Van der Schans CP: Inspiratory muscle training in patients with cystic fibrosis. Respir Med 2001; 95: 31-6.

15.- Sánchez $H$, Montemayor $T$, Ortega F, Cejudo $P$, et al: Inspiratory muscle training in patients with COPD: effect on dyspnea, exercise performance, and quality of life. Chest 2001; 120: 748-56.

16.- Yuan N, Skaggs DL, Dorey F, Keens TG: Preoperative predictors of prolonged postoperative mechanical ventilation in children following scoliosis repair. Pediatr Pulmonol 2005; 40: 414-9.

17.- Black LF, Hyatt RE: Maximal respiratory pressures: normal values and relationship to age and sex. Am Rev Respir Dis 1969; 99: 696-702.

18.- Szeinberg A, Marcotte JE, Roizin H, et al: Normal values of maximal inspiratory and expiratory pressures with a portable apparatus in children, adolescents, and young adults. Pediatr Pulmonol 1987; 3: 255-8.

19.- Hepper NGG, Black LF, Fowler WS: Relationships of lung volumes to height and arm span in normal subjects and in patients with spinal deformity. Am Rev Respir Dis 1965; 91: 356-62.

20.- Gutiérrez M, Rioseco F, Rojas A, Casanova D: Determinación de valores espirométricos en una población chilena normal mayor de 5 años, a nivel del mar. Rev Med Chile 1996; 124: 1295-306.

21.- Platzker ACG, Keens TG: Pulmonary function testing in pediatric patients. In: Wilson AF, editor. Pulmonary function testing: indications and interpretations. New York: Grune \& Stratton; 1985.

22.- Preis K, Swiatkowska-Freund M, Janczewska I: Spina bifida-a follow-up study of neonates born from 1991 to 2001. J Perinat Med 2005; 33: 353-6.

23.- Oakeshott P, Hunt G: Long-term outcome in open spina bifida. British Journal of General Practice 2003; 53: 632-6.

24.- Koessler W, Wanke T, Winkler G, Nader A, Toifl K: 2 Years' Experience With Inspiratory Muscle Training in Patients With Neuromuscular Disorders. Chest 2001; 120: 765-9.

25.- Topin N, Matecki S, Le Bris S, Ois Rivier F, Echenne B, Prefaut $C$, Ramonatxo $M$ : Dose-dependent effect of individualized respiratory muscle training in children with Duchenne muscular dystrophy. Neuromuscular Disorders 2002; 12: 576-83. 